Artículo

\title{
Impacto de cubierta, ecotipo y endomicorriza en morfología y calidad de chile piquín
}

\author{
Rosalinda Mendoza-Villarreal ${ }^{1}$ \\ Valentín Robledo-Torres ${ }^{1}$ \\ Miguel Ángel Pérez-Rodríguez ${ }^{1}$ \\ Reyna Roxana Guillén-Enríquez ${ }^{2}$ \\ Víctor Martínez-Cueto ${ }^{1}$ \\ José Rafael Paredes-Jácome ${ }^{3 \S}$ \\ ${ }^{1}$ Universidad Autónoma Agraria Antonio Narro. Buenavista, Saltillo, Coahuila, México. CP. 25315. \\ (rosalindamendoza@ hotmail.com; $\quad$ robledo3031@gmail.com; $\quad$ miguel_cbg@hotmail.com; \\ vimarc59@yahoo.com.mx). ${ }^{2}$ Tecnológico Nacional de México-Campus Instituto Tecnológico de Torreón. \\ Antigua Carretera Torreón-San Pedro km 7.5, Torreón, Coahuila, México. CP. 27170. \\ (reguillen@ outlook.com). ${ }^{3}$ Doctorado en Ciencias en Agricultura Protegida-Universidad Autónoma Agraria \\ Antonio Narro, Saltillo, Coahuila México. CP. 25315. \\ ${ }^{\S}$ Autor para correspondencia: rafael_2893@hotmail.com.
}

\section{Resumen}

La investigación se realizó con ecotipos de chile piquín de Coahuila, Nuevo León y Zacatecas. Se evaluaron plantas de chile piquín del segundo año de producción. Se utilizaron dos ambientes: a) macro túneles de malla blanca, roja azul, negra tipo raschel con $30 \%$ de sombreo; y b) campo abierto con $100 \%$ de transmisión de luz. Se inocularon 50 esporas de un conglomerado de hongos micorrízicos arbusculares (Glomus mosseae, Rhizophagus intraradices, Sclerocystis coremioides y Gigaspora albida), directamente al sistema radical. Se utilizó un arreglo factorial 5 x 6 x 2 (cubiertas, ecotipos y micorrizas) y su distribución fue un diseño bloques al azar con cuatro repeticiones. Se evaluó el rendimiento y algunos caracteres morfológicos como altura de planta (AP), diámetro de tallo (DT), longitud de raíz (LR), peso fresco de planta (PFP), peso fresco de planta (PSP), peso fresco de raíz (PFR), peso seco de raíz (PSR), rendimiento por planta (RPP). Los resultados indicaron que la malla blanca favoreció los caracteres morfológicos de la planta con $320 \%$ en AP, $322.7 \%$ en DT, $235.8 \%$ la LR, 8 veces el PFP, 8.5 veces el PSP, $327.2 \%$ el PFR, 5 veces el PSR, 6.8 veces el RPP, en comparación a las plantas desarrolladas en campo abierto. Además, la calidad de chile piquín proporciona condiciones para el desarrollo de endomicorrizas (esporas y porcentaje de colonización). La malla azul con la menor radiación fotosintéticamente activa (RFA) influyó positivamente en las variables agronómicas, de calidad y la inoculación (número de esporas y porcentaje de colonización). El ecotipo que influyó en los SST, VitC, fenoles y NE fue el de SNL y el RTZ en variables agronómicas y \% colonización. La inoculación con el consorcio de micorrizas mejoró los caracteres agronómicos y calidad del fruto de chile piquín.

Palabras clave: Capsicum annuum, calidad, malla sombra, radiación solar.

Recibido: enero de 2021

Aceptado: febrero de 2021 


\section{Introducción}

El chile piquín (Capsicum annuиm L.) es un recurso fitogenético ampliamente distribuido en México, dado su naturaleza la forma silvestre de esta especie es la que predomina, su fruto generalmente se obtiene por recolección en poblaciones silvestres, pero lo amenaza su diversidad genética (Pagán et al., 2010). El chile piquín no ha sido domesticado completamente ya que presenta una baja germinación y variabilidad morfológica y genética (García-Federico et al., 2010; Hernández-Verdugo et al., 2015).

Los rendimientos de chile piquín son afectados por las condiciones ambientales, humedad en el suelo y la fertilización (Rodríguez et al., 2005). En relación, a los factores ambientales se ha demostrado que la luz solar directa origina plantas más compactas, mientras que $80 \%$ de sombra las plantas crecen rápidamente y se vuelven más grandes. La respuesta de las plantas de Capsicum a diferentes condiciones de irradiancia puede variar según el cultivar. El fotoperiodo, calidad y cantidad de la luz solar coinciden, directamente, en la fotosíntesis de las plantas y demás características fenotípicas y funcionales (Peixoto et al., 2014).

Las respuestas fenotípicas de las plantas ante condiciones variadas de luz no han sido aprovechadas adecuadamente para modificar las características morfo-fisiológicas de los cultivos y obtener un rendimiento y calidad deseada (Kelly et al., 2015). El uso de mallas de colores es una alternativa para evitar la excesiva radiación como sucede con la malla roja, que proporciona $42.6 \%$ radiación solar total (350 a $1050 \mathrm{~nm}$ ) y la malla azul $36 \%$ más luz azul $(400$ a $500 \mathrm{~nm}$ ) que la malla negra (Ayala-Tafoya et al., 2015). Estas diferencias en la radiación pueden causar respuesta diferencial en la fotosíntesis y fotomorfogénesis que producen efectos sobre el crecimiento del tallo, expansión foliar, desarrollo de cloroplastos, síntesis de clorofila y metabolitos secundarios. En pepino el uso de mallas de color rojo y azul incrementaron el rendimiento 48.1 y $46.1 \%$ respecto al testigo (Ayala-Tafoya et al., 2015).

Por otro lado, el uso de biofertilizantes en el cultivo de chile piquín ha demostrado efectos positivos en altura de planta, longitud de raíz, mayor biomasa seca en suelos salinos y no salinos (Rueda et al., 2010). También se ha demostrado que los hongos micorrízicos presentan afinidad en diferentes especies de Capsicum como annuum, bacatum, chinense, frutescens y pubescens lo que confirma su efecto positivo (Cardona et al., 2008).

Sin embargo, la aplicación de inóculos comerciales no asegura el efecto positivo en el sinergismo planta-hongo micorrízico, debido al uso indiscriminado de agroquímicos en los sistemas de producción tradicional (Koyama et al., 2017; Caruso et al., 2018). Además, en otros cultivos como el tomate se han utilizado micorrizas (Glomus mossae y Glomus cubense) en forma líquida (10 y 20 esporas) y sólida (20 y 40 esporas) y han producido resultados benéficos para la planta (Mujica et al., 2012).

El objetivo del presente trabajo de investigación fue evaluar el efecto de la inoculación con endomicorrizas nativas de tres localidades del noreste del país bajo cubiertas fotoselectivas y campo abierto en la morfología y calidad de chile piquín. 


\section{Materiales y métodos}

El estudio se realizó, en el Departamento de Horticultura de la Universidad Autónoma Agraria Antonio Narro, Saltillo, Coahuila, México, ubicada a $25^{\circ} 22^{\prime}$ latitud norte y $101^{\circ} 22^{\prime}$ longitud oeste, a una altura de $1580 \mathrm{msnm}$. Se colectaron seis ecotipos de chile piquín, denominados: MZC= Múzquiz, Coahuila; SAC= San Alberto, Coahuila; LNL= Linares, Nuevo León; SNL= Santiago, Nuevo León; PTZ= Puente Tepetatilla, Zacatecas y RTZ= Río Tuxpan, Zacatecas.

La siembra de semillas se realizó en cajas germinadoras a las cuales se les aplicó GA3 (500ppm) para acelerar el proceso de germinación, después de un mes se trasplantaron las plántulas en bolsas de polietileno con capacidad de $10 \mathrm{~L}$ y se colocaron a una distancia de $40 \mathrm{~cm}$ entre plantas y $1 \mathrm{~m}$ entre surcos, como sustrato se utilizó turba de sphagnum (Pro Mix ${ }^{\circledR}$ ) y perlita $\left(\right.$ Hortiperl $^{\circledR}$ ) en una proporción 2:1 (v/v). En el experimento se utilizaron estos ecotipos de chile en su segundo año de producción, se utilizaron distintos ambientes de luminosidad (color de cubierta): a) macro túneles de malla blanca (MA), roja (MR), azul (MA), negra (MN) tipo raschel con 30\% de sombreo, con un tamaño de orificio de 6 × $8 \mathrm{~mm}$; cada túnel de $4 \mathrm{~m}$ de ancho, $6 \mathrm{~m}$ de largo y $2.3 \mathrm{~m}$ de alto; y b) campo abierto con el $100 \%$ de transmisión de luz.

El cultivo se fertilizó con solución nutritiva, al 25\% en plántula, al 50\% en desarrollo vegetativo, $75 \%$ en floración y $100 \%$ en fructificación. Al comienzo de esta evaluación se disminuyó el aporte de fósforo a $25 \%$, con el fin de que las endomicorrizas presentaran sinergia con las raíces de las plantas. El aporte de agua se realizó de 0.5 a $2.5 \mathrm{~L} \mathrm{planta}^{-1}$ día $^{-1}$, con un sistema de fertirriego por estacas.

Las plantas se inocularon después del trasplante con (50 esporas) con un conglomerado de hongos micorrízicos arbusculares (Glomus mosseae, Rhizophagus intraradices, Sclerocystis coremioides y Gigaspora albida), directamente al sistema radical, los cuáles fueron identificados mediante morfología comparada (Sánchez-Sánchez et al., 2018).

Estos factores fueron evaluados mediante un arreglo factorial $5 \times 6 \times 2$ (color de cubiertas:5, ecotipos:6 y micorriza:2). El diseño experimental utilizado correspondió a un bloque al azar con cuatro repeticiones. Las variables microclimáticas cómo temperatura ambiental y humedad relativa fueron registradas con un termo higrómetro digital (Taylor $^{\circledR}$, modelo 1452). La radiación fotosintéticamente activa (RFA) se registró con un sensor portátil Quantum (Apogee ${ }^{\circledR}$, modelo SM700). Las mediciones fueron realizadas diariamente entre las 07:00 y 19:00 h, en el centro de cada cubierta, en condiciones de cielo despejado.

Los caracteres morfológicos se evaluaron en tres plantas por repetición y tratamiento, los cuáles incluyeron: altura de planta (AP), realizada con cinta métrica, diámetro de tallo (DT), con un vernier digital (Digital Caliper ${ }^{\circledR}$ ), se realizaron ocho cortes, para obtener el rendimiento promedio de fruto por planta, se pesaron con una báscula electrónica Rhino modelo Babol-100G con capacidad de $100 \mathrm{~g}$ y resolución de $0.01 \mathrm{~g}$. El peso fresco (PFP) y seco (PSP) de planta y de raíz (PFR) se determinó en una báscula OHAUS modelo CS-5000 con capacidad de $5 \mathrm{~kg}$. Para obtener el peso seco de planta (PSP) y de raíz (PSR), las muestras fueron colocadas en papel de estraza y sometidas a $65{ }^{\circ} \mathrm{C}$ durante $48 \mathrm{~h}$ en una estufa de secado Yamato modelo DX-602 y posteriormente se pesaron en la báscula antes mencionada. 
Se estimó el número de hojas (NH), el número de frutos por planta (NFPP), mediante el recuento en cada unidad, las evaluaciones se hicieron en todas las unidades experimentales. En variables de calidad se evaluaron tres muestras por tratamiento y repetición para, sólidos solubles totales (SST) con un refractómetro digital HANNA 96-801, en el cual se colocó una gota de pulpa de fruto en la celda del aparato, obteniendo el contenido expresado en Brix. El contenido de ácido ascórbico (Vitamina C), en frutos se determinó por la metodología de la AOAC (2000).

El contenido de fenoles totales (FT) fue determinado de acuerdo a la metodología reportada por Kim et al. (2006), con algunas modificaciones las cuales se describen a continuación se pesaron 2 $\mathrm{g}$ de fruto de chile piquín fresco y se colocaron en $20 \mathrm{ml}$ de metanol al $80 \%$, durante $12 \mathrm{~h}$ a $4{ }^{\circ} \mathrm{C}$, transcurrido el tiempo se centrifugó a $12000 \mathrm{rpm}$ durante $5 \mathrm{~min}$, se tomó una alícuota de $200 \mu \mathrm{l}$ del sobrenadante mezclado con $150 \mu \mathrm{l}$ del agente Folin Ciocaltaeu $2 \mathrm{~N}$, se le añadieron $2 \mathrm{ml}$ de $\mathrm{Na}_{2} \mathrm{CO}_{3}$ al 2\%, dejándolo incubar por 25 min y finalmente se leyó la absorbancia a $735 \mathrm{~nm}$ en espectrofotómetro (Bio-145025 BIOMATE 5 Thermo elctron Corporation), la curva de calibración se realizó con ácido gálico.

La cuantificación de capsaicina (CAPs) fue determinada en frutos con madurez fisiológica, por el método descrito por Bennet y Kirby (1965), mediante un espectrofotómetro (Bio-145025 Biomate5 Thermo Electron Corporation) a una longitud de onda de $286 \mathrm{~nm}$, en la cual la capsaicina se encuentra en su fase orgánica. Para la determinación de la concentración se construyó una curva de calibración de este antioxidante (Sigma, Co) en un rango 0.5 a $1.5 \mathrm{mg} \mathrm{ml}^{-1}$. En variables de calidad las evaluaciones fueron por triplicado para cada tratamiento.

El número de esporas fue cuantificado por triplicado para cada tratamiento y repetición en $100 \mathrm{~g}$ de suelo con el método de tamizado húmedo y decantación (Genderman y Nicolson, 1963), la limpieza y tinción de raíces se realizó con el método de Phillips y Hayman (1970) y la colonización (McGonigle, 1990) la cual consiste en lavar las raíces con agua corriente, cortarlas y colocarlas en tubos falcón de $25 \mathrm{ml}$, cubrirlas con $\mathrm{KOH}$ al $10 \%$ por $24 \mathrm{~h}$ a temperatura ambiente, posteriormente se enjuagaron con abundante agua corriente, se cubrieron con $\mathrm{H}_{2} \mathrm{O}_{2}$, durante 5 min, después se enjuagaron con agua corriente, se cubrieron las raíces con $\mathrm{HCl}$ al 10\%, por $10 \mathrm{~min}$, posteriormente se decantó el $\mathrm{HCl}$ y sin enjuagar las raíces, se les añadió la solución azul de tripano al $0.05 \%$ por $24 \mathrm{~h}$ a temperatura ambiente, transcurrido el tiempo se eliminó el colorante con ayuda de un tamiz y se colocaron en lactoglicerol, finalmente se cortaron segmentos de $1 \mathrm{~cm}$ de raíz y se depositaron en un portaobjetos, se cubren y se observan en un microscopio óptico (Axio Scope A1, Carl Zeiss, Microscopy $\mathrm{GmbH}$, Gotting, Germany). Los resultados obtenidos fueron analizados mediante un análisis de varianza y la comparación de medias por la prueba de Tukey $(p \leq 0.05)$, utilizándose el programa estadístico SAS versión 9.0.

\section{Resultados y discusión}

En la Figura 1 se midió la RFA observándose que la máxima radiación se encuentra entre 13 y 15 h, y en CA se presenta 95\% mayor comparada con la MR quién capta una radiación entre 350 a 1 $050 \mathrm{~nm}$, con muy parecido comportamiento la MB y MN y la menor absorción se llevó a cabo con la MA cuya radiación se encuentra entre 400 y $500 \mathrm{~nm}$ (Ayala-Tafoya et al., 2015). En cultivos de flores la malla negra reduce la RFA entre 55 y $60 \%$ dependiendo de la estación del año y la malla roja de 41 al 51\% (Arthurs et al., 2013). 


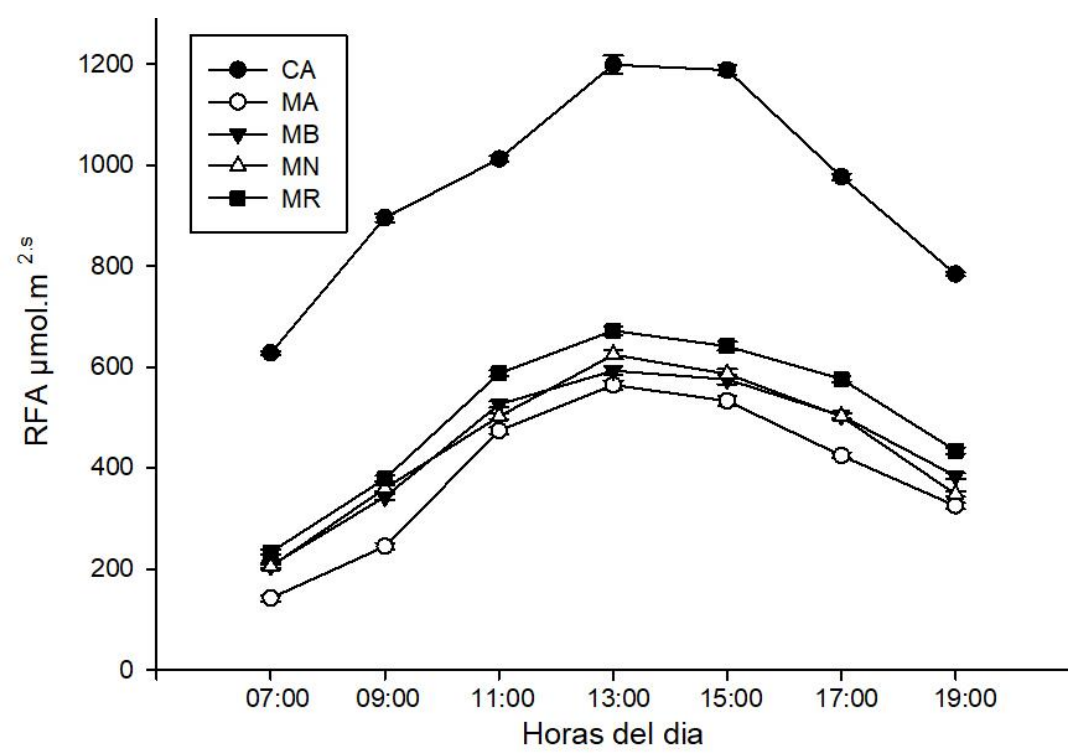

Figura 1. Radiación fotosintéticamente activa en campo abierto y mallas de colores en plantas de chile piquín.

En el Cuadro 1 se muestran los valores de temperatura, siendo el mes con mayor registro el de mayo en campo abierto disminuyendo entre 1 y 2 grados hasta el mes de septiembre. También se observa que la MR que es muy similar en la temperatura comparada con CA y la MA disminuye de 2 a 5 grados en relación, a CA. En el mismo Cuadro 1 se observa que la HR se incrementó cada mes hasta llegar a su máximo en el mes de septiembre, también en CA se observan las mayores HR solo variando en septiembre con MA y MB.

Cuadro 1. Valores promedios mensuales de las variables climáticas monitoreadas en cada condición ambiental bajo campo abierto y mallas de colores.

\begin{tabular}{ccccccc}
\hline \multirow{2}{*}{ Cubierta } & Variable & \multicolumn{5}{c}{ Meses evaluados } \\
\cline { 3 - 7 } & & Mayo & Junio & Julio & Agosto & Septiembre \\
\hline CA & $\mathrm{T}\left({ }^{\circ} \mathrm{C}\right)$ & 26.98 & 25.56 & 23.34 & 22.86 & 20.74 \\
& $\mathrm{HR}(\%)$ & 34.54 & 43.1 & 57.32 & 62.38 & 69.36 \\
MA & $\mathrm{T}\left({ }^{\circ} \mathrm{C}\right)$ & 21.45 & 22.34 & 21.55 & 19.33 & 18.44 \\
& $\mathrm{HR}(\%)$ & 34.22 & 42.11 & 55.32 & 63.44 & 70.44 \\
MB & $\mathrm{T}\left({ }^{\circ} \mathrm{C}\right)$ & 22.13 & 23.11 & 22.11 & 20.55 & 18.98 \\
& $\mathrm{HR}(\%)$ & 34.58 & 44.55 & 55.67 & 61.78 & 65.89 \\
\multirow{2}{*}{ MN } & $\mathrm{T}\left({ }^{\circ} \mathrm{C}\right)$ & 22.33 & 22.41 & 22.15 & 20.77 & 20.11 \\
& $\mathrm{HR}(\%)$ & 33.77 & 42.33 & 55.65 & 60.43 & 66.51 \\
& $\mathrm{~T}\left({ }^{\circ} \mathrm{C}\right)$ & 23.78 & 24.51 & 23.11 & 21.52 & 20.17 \\
& $\mathrm{HR}(\%)$ & 33.11 & 41.22 & 56.73 & 60.22 & 68.55 \\
\hline
\end{tabular}

$\mathrm{T}=$ temperatura; $\mathrm{HR}=$ humedad relativa. 
En el Cuadro 2 se registra que la MB favorece positivamente las variables agronómicas evaluadas con 320\% en AP, 322.7\% en DT, 235.8\% la LR, 8 veces el PFP, 8.5 veces el PSP, 327.2\% el PFR, 5 veces el PSR, 6.8 veces el RPP y 4.8 veces el NFPP comparadas con CA que fue el ambiente menos favorable, quizá en condiciones normales de crecimiento de plantas de chile piquín estas se encuentran bajo la sombra de un árbol o arbusto lo que impide la radiación directa. La MB refleja $70 \%$ de radiación permitiendo condiciones favorables para el crecimiento de la planta, la malla color perla aumentó su transmisión desde los $700 \mathrm{~nm}$ lo que favorece la fotosíntesis del pepino (Ayala-Tafoya et al., 2015) y en consecuencia el crecimiento de la planta a diferencia de lo reportado por Shahak et al. (2006) indicando que la producción se incrementó en tres cultivares de chile pimiento con la malla perla $16 \%$ y con malla roja un $32 \%$ en comparación con malla negra.

El ecotipo que favoreció las variables antes mencionadas fue RTZ, solo LNL incrementó la LR y MZC el PSR (Cuadro 2). Sin embargo, se ha reportado que cada morfotipo presenta diferente a las condiciones ambientales como sucede con los morfotipos de Oaxaca cuyas características fenotípicas son muy diferentes (Castellón Martínez et al., 2014), a las de Nuevo León, Coahuila y Zacatecas utilizadas en este estudio. En el mismo Cuadro 2, se observa que la inoculación con micorrizas nativas tuvo respuesta favorable con el incremento de $53 \%$ en AP, en $32 \%$ DT, en 70.24\% PFP, en $66.9 \%$ PSP, en R 72.2\% y en NFPP en $28.8 \%$. Lo que coincide con Cardona et al. (2008) quienes encontraron que las micorrizas arbusculares colonizan las raíces del género Capsicum, por eso se mejoraron los caracteres morfológicos de chile piquín.

Cuadro 2. Variables agronómicas de los diferentes factores evaluados en plantas de chile piquín.

\begin{tabular}{|c|c|c|c|c|c|c|c|c|c|}
\hline Cubierta & $\begin{array}{l}\text { AP } \\
(\mathrm{cm})\end{array}$ & $\begin{array}{c}\text { DT } \\
(\mathrm{mm})\end{array}$ & $\begin{array}{l}\text { LR } \\
(\mathrm{cm})\end{array}$ & $\begin{array}{c}\text { PFP } \\
(\mathrm{g})\end{array}$ & $\begin{array}{c}\text { PSP } \\
(\mathrm{g})\end{array}$ & $\begin{array}{c}\text { PFR } \\
(\mathrm{g})\end{array}$ & $\begin{array}{c}\text { PSR } \\
(\mathrm{g})\end{array}$ & $\begin{array}{c}\text { RPP } \\
(\mathrm{g})\end{array}$ & NFPP \\
\hline $\mathrm{CA}$ & $22.94 \mathrm{e}$ & $4.1 f$ & $15.1 \mathrm{e}$ & $24.81 \mathrm{e}$ & $11.34 \mathrm{e}$ & $10.6 \mathrm{e}$ & $4.36 \mathrm{e}$ & $5.5 f$ & $36.52 \mathrm{f}$ \\
\hline $\mathrm{MN}$ & $44.62 d$ & $6.96 \mathrm{e}$ & $28.44 d$ & $43.04 d$ & $19.56 \mathrm{e}$ & $18.13 d$ & $7.21 \mathrm{~d}$ & $16.81 \mathrm{~d}$ & $80.1 \mathrm{e}$ \\
\hline MR & $53.69 \mathrm{~b}$ & $9.76 b$ & $29.79 \mathrm{c}$ & $93.63 b$ & $36.59 b$ & $21.14 \mathrm{c}$ & $11.74 \mathrm{c}$ & $18.08 \mathrm{c}$ & $113.08 b$ \\
\hline MA & $47.25 \mathrm{~cd}$ & $9.39 \mathrm{c}$ & $36.12 \mathrm{a}$ & $64.13 c$ & $29.31 \mathrm{c}$ & $24.31 b$ & $14.54 \mathrm{~b}$ & $22.8 b$ & $105.17 \mathrm{c}$ \\
\hline $\mathrm{MB}$ & $73.42 \mathrm{a}$ & $13.23 \mathrm{a}$ & $35.6 \mathrm{ab}$ & $200.52 \mathrm{a}$ & $96.71 \mathrm{a}$ & $34.68 \mathrm{a}$ & $21.32 \mathrm{a}$ & $37.42 \mathrm{a}$ & $177.08 \mathrm{a}$ \\
\hline \multicolumn{10}{|c|}{ Ecotipo } \\
\hline SAC & $33.75 \mathrm{e}$ & $6.9 \mathrm{e}$ & $28.22 \mathrm{e}$ & $44.85 d$ & $20.89 \mathrm{e}$ & $19.45 \mathrm{f}$ & $10.75 d$ & $7.09 \mathrm{f}$ & $59.5 \mathrm{f}$ \\
\hline $\mathrm{MZC}$ & $42.35 \mathrm{~d}$ & $8.46 \mathrm{c}$ & $28.28 \mathrm{~d}$ & $76.44 \mathrm{c}$ & $34.71 \mathrm{~cd}$ & $24.37 b$ & $14.53 \mathrm{a}$ & $26.08 b$ & $133.75 b$ \\
\hline PTZ & $44.6 \mathrm{~cd}$ & $8.73 c$ & $27.8 \mathrm{f}$ & $75.63 \mathrm{c}$ & $32.01 \mathrm{~d}$ & $20.81 \mathrm{c}$ & $11.24 \mathrm{c}$ & $10.57 d$ & $70.9 \mathrm{e}$ \\
\hline RTZ & $71.25 \mathrm{a}$ & $9.8 \mathrm{a}$ & $29.13 c$ & $115.54 \mathrm{a}$ & $48.73 a$ & $20.7 d$ & $10.35 \mathrm{f}$ & $50.48 \mathrm{a}$ & $169.31 \mathrm{a}$ \\
\hline LNL & $52.46 \mathrm{~b}$ & $9.29 b$ & $30.68 \mathrm{a}$ & $87.44 b$ & $43.87 b$ & $25.15 \mathrm{a}$ & $13.43 b$ & $11.93 \mathrm{c}$ & $94.19 \mathrm{c}$ \\
\hline SNL & $46.08 \mathrm{c}$ & $7.68 \mathrm{~d}$ & $29.73 b$ & $74.94 c$ & $37.16 \mathrm{c}$ & $20.15 \mathrm{e}$ & $10.7 \mathrm{e}$ & $9.27 \mathrm{e}$ & $78.1 \mathrm{~d}$ \\
\hline \multicolumn{10}{|c|}{ Consorcio } \\
\hline SM & $38.28 b$ & $7.31 b$ & $25.25 b$ & $58.57 \mathrm{~b}$ & $27.15 b$ & 18.03 & $9.05 b$ & $14.13 b$ & $88.24 b$ \\
\hline $\mathrm{CM}$ & $58.56 \mathrm{a}$ & $9.65 a$ & $32.69 \mathrm{a}$ & $99.71 \mathrm{a}$ & $45.3 \mathrm{a}$ & $25.52 \mathrm{a}$ & $14.62 \mathrm{a}$ & $24.33 \mathrm{a}$ & $113.68 \mathrm{a}$ \\
\hline $\mathrm{S}$ & . & 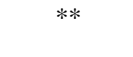 & ? & (n) & 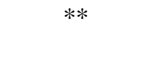 & *** & 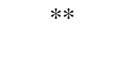 & . & . \\
\hline $\mathrm{CV}(\%)$ & 9.53 & 5.82 & 8.54 & 13.78 & 14.59 & 9.91 & 14.86 & 5.89 & 5.81 \\
\hline
\end{tabular}

$\mathrm{AP}=$ altura de planta; $\mathrm{DT}=$ diámetro de tallo; $\mathrm{LR}=$ longitud de raíz; $\mathrm{PFP}=$ peso fresco de planta; $\mathrm{PSP}=$ peso seco de planta; $\mathrm{PFR}=$ peso fresco de raíz; $\mathrm{PSR}=$ peso seco de raíz; $\mathrm{RPP}=$ rendimiento por planta; $\mathrm{NFP}=$ número de frutos por planta; RTZ= Río Tuxpan, Zacatecas; PTZ= Puente Tepetatilla, Zacatecas; LNL= Linares, Nuevo León; MZC= Múzquiz, Coahuila; SNL= Santiago, Nuevo León; SAC= San Alberto, Coahuila; MB= malla blanca; MA= malla azul; $\mathrm{MR}=$ malla roja; $\mathrm{MN}=$ malla negra $; \mathrm{CA}=$ campo abierto; $\mathrm{SM}=$ sin micorriza; $\mathrm{CM}=$ con micorriza. Medias con la misma letra dentro de cada columna en cada factor no difieren estadísticamente (Tukey, $p \leq 0.05$ ). CV= coeficiente de variación. $\mathrm{S}=$ significancia; ${ }^{* *}=$ altamente significativo $(p \leq 0.001)$. 
En el Cuadro 3, se presentan las variables de calidad, se observa que para SST se incrementan en frutos con $32 \%$ con la MR y $37.6 \%$ en MB, la vitamina C $(42.9 \%)$ y fenoles totales también con (44.31\%) la MB y Capsaicina con MA $18.42 \%$ además del picor representado por unidades Scoville (SHU) en la MA, en el mismo Cuadro 3 los ecotipos que presentan diferencias estadísticas para SST son los ecotipo MZC y SNL. Para Vitamina C, el SAC y SNL, fenoles totales el SNL, para capsaicina y picor (US) el PTZ.

Cuadro 3. Variables de calidad de los factores evaluados en plantas de chile piquín.

\begin{tabular}{|c|c|c|c|c|c|}
\hline Cubierta & SST ( ${ }^{\circ}$ Brix) & $\operatorname{VitC}\left(\mathrm{mg} 100 \mathrm{~g}^{-1}\right)$ & $\mathrm{FT}\left(\mu \mathrm{g}\right.$ EAG $\left.\mathrm{g}^{-1}\right)$ & CAPs $\left(\mathrm{mg} \mathrm{g}^{-1}\right)$ & $\begin{array}{c}\text { U Scoville } \\
\text { SHU }\end{array}$ \\
\hline $\mathrm{CA}$ & $6.41 \mathrm{c}$ & $60.85 f$ & $34.77 \mathrm{e}$ & $2.66 \mathrm{~d}$ & $42560 d$ \\
\hline $\mathrm{MN}$ & $6.69 c$ & $66.3 \mathrm{e}$ & $37.09 d$ & $3.07 \mathrm{~b}$ & $49120 b$ \\
\hline MR & $8.46 \mathrm{a}$ & $84.29 b$ & $42.42 b$ & $2.95 \mathrm{c}$ & $47200 c$ \\
\hline MA & $7.52 b$ & $71.24 \mathrm{c}$ & $43.05 b$ & $3.15 \mathrm{a}$ & $50400 \mathrm{a}$ \\
\hline MB & $8.82 \mathrm{a}$ & $86.96 a$ & $50.18 \mathrm{a}$ & $3.09 \mathrm{ab}$ & $49440 \mathrm{ab}$ \\
\hline \multicolumn{6}{|c|}{ Ecotipo } \\
\hline SAC & $6.71 c$ & $82.98 \mathrm{a}$ & $34.91 \mathrm{~d}$ & $2.98 \mathrm{bc}$ & $47680 \mathrm{bc}$ \\
\hline $\mathrm{MZC}$ & $8.57 \mathrm{a}$ & $68 d$ & $37.47 \mathrm{c}$ & $2.93 \mathrm{c}$ & $46880 c$ \\
\hline PTZ & $8.31 \mathrm{a}$ & $79.95 b$ & $37.54 \mathrm{c}$ & $3.09 \mathrm{a}$ & $49440 \mathrm{a}$ \\
\hline RTZ & $7.4 \mathrm{~b}$ & $78.38 \mathrm{c}$ & $46.28 b$ & $3.02 \mathrm{~b}$ & $48320 b$ \\
\hline LNL & $7.41 \mathrm{~b}$ & $82.97 \mathrm{a}$ & $35.82 d$ & $2.93 \mathrm{c}$ & $46880 c$ \\
\hline SNL & $8.39 \mathrm{a}$ & $45.21 \mathrm{e}$ & $55.37 \mathrm{a}$ & $3.02 \mathrm{~b}$ & $48320 b$ \\
\hline \multicolumn{6}{|c|}{ Consorcio } \\
\hline $\mathrm{SM}$ & $8.31 \mathrm{a}$ & $72.27 b$ & $40.08 b$ & $2.92 b$ & $46720 b$ \\
\hline $\mathrm{CM}$ & $7.29 b$ & $73.56 \mathrm{a}$ & $42.38 \mathrm{a}$ & $3.07 \mathrm{a}$ & $49120 \mathrm{a}$ \\
\hline $\mathrm{S}$ & $* *$ & $* *$ & $* *$ & $* *$ & $* *$ \\
\hline $\mathrm{CV}(\%)$ & 5.09 & 0.85 & 5.79 & 3.98 & 3.98 \\
\hline
\end{tabular}

SST = sólidos solubles totales; Vit $\mathrm{C}=$ vitamina $\mathrm{C}$; FT= fenoles totales; $\mathrm{CAPs}=$ capsaicina; $\mathrm{RTZ}=$ Río Tuxpan, Zacatecas; PTZ= Puente Tepetatilla, Zacatecas; LNL= Linares, Nuevo León; MZC= Múzquiz, Coahuila; SNL= Santiago, Nuevo León; SAC= San Alberto, Coahuila; MB= malla blanca; $M A=$ malla azul; $M R=$ malla roja; $\mathrm{MN}=$ malla negra; $\mathrm{CA}=$ campo abierto; $\mathrm{SM}=$ sin micorriza; $\mathrm{CM}=$ con micorriza. Medias con la misma letra dentro de cada columna en cada factor no difieren estadísticamente (Tukey, $p \leq 0.05$ ). $\mathrm{CV}=$ coeficiente de variación. $\mathrm{S}=$ significancia; $*^{* *}=$ altamente significativo $(p \leq 0.001)$.

La aplicación de CM produjo mejores resultados en vitamina C (1\%), fenoles totales (3.87\%) y capsaicina $(5.13 \%)$ incrementando los antioxidantes del fruto y el picor, solo en SST se obtuvo el $27 \%$ de incremento SM. En relación, a la calidad se realizó un estudio con diferentes morfotipos de chile como piquín y solterito los cuáles produjeron mayor contenido de fenoles y flavonoides además de capsaicina (Wei et al., 2013), aunque no se añadieron micorrizas la tendencia de los morfotipos es producir mayor contenido de antioxidantes. 
En el Cuadro 4, se encontró que al analizar por separado la cubierta la MB es la que incrementa en 96\% el número de esporas y 101.4 el \% de colonización. También se muestra que el ecotipo SNL incrementó el NE y RTZ el \% col y en relación, a la inoculación con micorrizas nativas se favoreció el NE y \% col en plantas de chile piquín. Esto concuerda con un estudio realizado en papaya al inocular con Glomus sp encontrando un incremento del porciento de colonización (QuiñonesAguilar et al., 2014).

Cuadro 4. Variables microbiológicas de los factores evaluados en plantas de chile piquín.

\begin{tabular}{ccc}
\hline Cubierta & NE & Col (\%) \\
\hline CA & $23.33 \mathrm{e}$ & $16.17 \mathrm{c}$ \\
MN & $36.67 \mathrm{~b}$ & $23.67 \mathrm{~b}$ \\
MR & $31.15 \mathrm{~cd}$ & $25.67 \mathrm{~b}$ \\
MA & $35.83 \mathrm{bc}$ & $25.92 \mathrm{~b}$ \\
MB & $45.73 \mathrm{a}$ & $32.57 \mathrm{a}$ \\
& Ecotipo & \\
SAC & $29.27 \mathrm{c}$ & $25.58 \mathrm{ab}$ \\
MZC & $34.48 \mathrm{ab}$ & $25.42 \mathrm{ab}$ \\
PTZ & $34.79 \mathrm{ab}$ & $23.42 \mathrm{~b}$ \\
RTZ & $35 \mathrm{ab}$ & $27.58 \mathrm{a}$ \\
LNL & $31.56 \mathrm{bc}$ & $23.5 \mathrm{~b}$ \\
SNL & $37.19 \mathrm{a}$ & $25.08 \mathrm{~b}$ \\
& Consorcio & \\
SM & $5.38 \mathrm{~b}$ & $2.31 \mathrm{~b}$ \\
CM & $62.05 \mathrm{a}$ & $47.89 \mathrm{a}$ \\
S & $*$ & 23.62 \\
CV $(\%)$ & 25.75 & . \\
\hline
\end{tabular}

$\mathrm{NE}=$ número de esporas; $\mathrm{Col}=$ porcentaje de colonización; $\mathrm{RTZ}=$ Río Tuxpan, Zacatecas; $\mathrm{PTZ}=$ Puente Tepetatilla, Zacatecas; LNL= Linares, Nuevo León; MZC= Múzquiz, Coahuila; SNL= Santiago, Nuevo León; SAC = San Alberto, Coahuila; $\mathrm{MB}=$ malla blanca; $\mathrm{MA}=$ malla azul; $\mathrm{MR}=$ malla roja; $\mathrm{MN}=$ malla negra; $\mathrm{CA}=$ campo abierto; $\mathrm{SM}=\sin$ micorriza; $\mathrm{CM}=$ con micorriza. Medias con la misma letra dentro de cada columna en cada factor no difieren estadísticamente (Tukey, $p \leq 0.05$ ).

El análisis de componentes principales (ACP), realizado para el tipo de cubierta y variables evaluadas Figura 3, mostró que la MB influyó positivamente en las variables RPP, PFR, AP, PSR, DT y NFPP, caso contrario a lo que se obtuvo con la MN y CA, mientras que la MA influyó en el contenido de Caps y LR, a su vez la MR promovió un aumentó en las variables Vit C, SST, PFP y PSP, por lo cual el uso de diferentes cubiertas propician resultados diversos en morfología y calidad en plantas de chile piquín; concordando con lo reportado en pepino (Ayala-Tafoya et al., 2015) al trabajar con mallas de colores. 


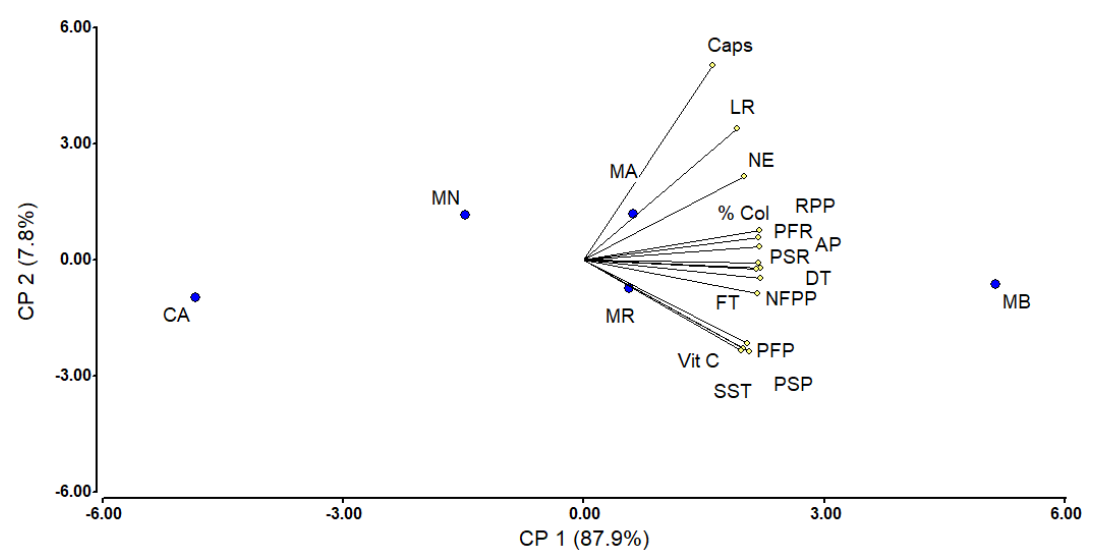

Figura 3. Componentes principales de las variables analizadas y tipo de cubierta. $A P=$ altura de planta; $\mathrm{DT}=$ diámetro de tallo; $\mathrm{LR}=$ longitud de raíz; $\mathrm{PFP}=$ peso fresco de planta; $\mathrm{PSP}=$ peso seco de planta; $\mathrm{PFR}=$ peso fresco de raíz; $\mathrm{PSR}=$ peso seco de raíz; $\mathrm{RPP}=$ rendimiento por planta; $\mathrm{NFP}=$ número de frutos por planta; $\mathrm{SST}=$ sólidos solubles totales; Vit $\mathrm{C}=$ vitamina $\mathrm{C}$; $\mathrm{FT}=$ fenoles totales; $\mathrm{CAPs}=$ capsaicina; $\mathrm{NE}=$ número de esporas; $\% \mathrm{Col}=$ porcentaje de colonización; $\mathrm{MB}=$ malla blanca; $\mathrm{MA}=$ malla azul; $\mathrm{IN}=$ invernadero; $\mathrm{MR}=$ malla roja; $\mathrm{MN}=$ malla negra; $\mathrm{CA}=$ campo abierto.

El ACP para ecotipos y variables evaluadas Figura 4, reveló un comportamiento disperso de los ecotipos evaluados; sin embargo, resalta que el ecotipo RTZ favorece las variables AP, PSP, RPP, NFPP, DT; mientras que los ecotipos SAC y PTZ no se ven favorecidos en esas variables, mientras que el ecotipo LNL favoreció el aumento en el PFR y PSR y a su vez los ecotipos MZC y SNL influyeron en las variables de calidad, Vit C, Caps y SST, lo que demuestra que el ecotipo juega un papel primordial en las variables que se evaluaron. Esto concuerda con Wei et al. (2013) al trabajar con diferentes morfotipos de chile en donde encuentra diferencias en el contenido de capsaicina y fenoles.

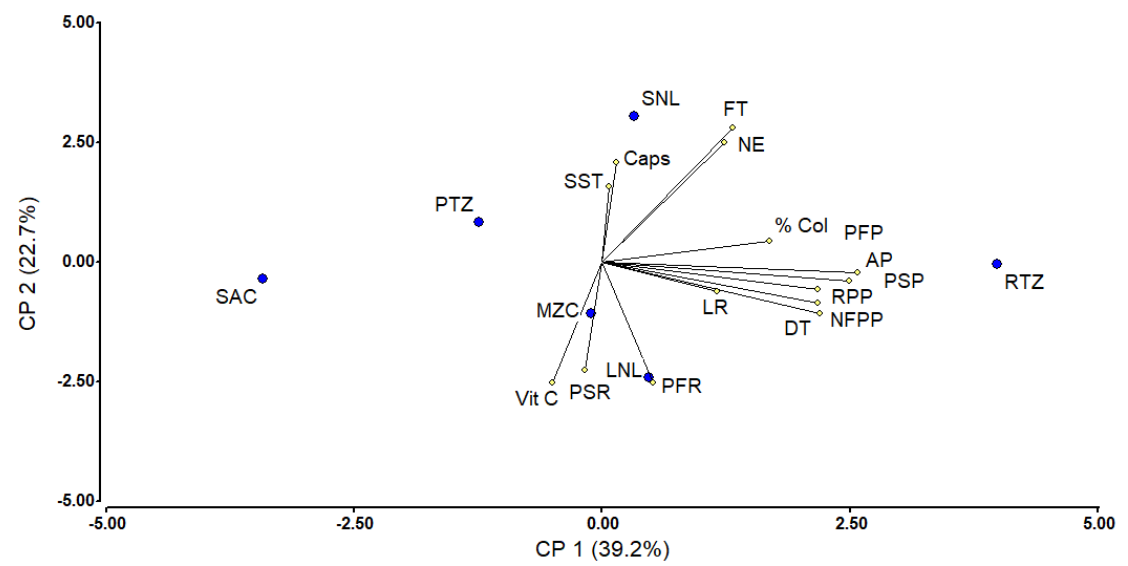

Figura 4. Componentes principales de las variables analizadas y el ecotipo. $\mathrm{AP}=$ altura de planta; $\mathrm{DT}=$ diámetro de tallo; $\mathrm{LR}=$ longitud de raíz; $\mathrm{PFP}=$ peso fresco de planta; $\mathrm{PSP}=$ peso seco de planta; $\mathrm{PFR}=$ peso fresco de raíz; $\mathrm{PSR}=$ peso seco de raíz; $\mathrm{RPP}=$ rendimiento por planta; $\mathrm{NFPP}=$ número de frutos por planta; SST= solidos solubles totales; Vit $\mathrm{C}=$ vitamina $\mathrm{C}$; FT= fenoles totales; $\mathrm{CAPs}=$ capsaicina; $\mathrm{NE}=$ número de esporas; $\% \mathrm{Col}=$ porcentaje de colonización; $\mathrm{RTZ}=$ Río Tuxpan, Zacatecas; PTZ= Puente Tepetatilla, Zacatecas; LNL= Linares, Nuevo León; MZC= Múzquiz, Coahuila; SNL=Santiago, Nuevo León; SAC=San Alberto, Coahuila. 
El ACP con la aplicación de micorrizas (CM) y la ausencia de estas (SM) con las variables estudiadas en la Figura 5, demuestra que hubo una fuerte relación en la mayoría de las variables evaluadas cuando se inocularon con micorrizas (Glomus mosseae, Rhizophagus intraradices, Sclerocystis coremioides y Gigaspora albid), a excepción de Vit C y de la variable SST que se incrementó cuando no se aplicaron las micorrizas. Resultados similares se obtuvieron en tomate cuando se inoculó con Glomus mossae y G. cubense con 20 y 40 esporas al incrementar el rendimiento (Mujica, 2012).

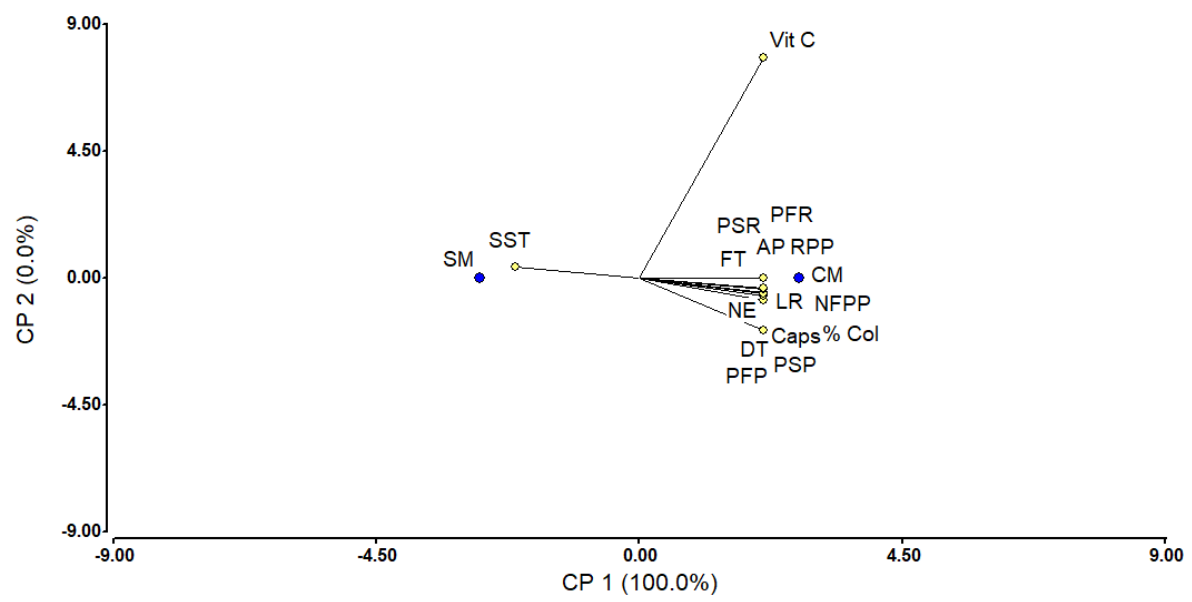

Figura 5. Componentes principales de las variables analizadas y Consorcio. $A P=$ altura de planta; $D=$ diámetro de tallo; $\mathrm{LR}=$ longitud de raíz; $\mathrm{PFP}=$ peso fresco de planta; $\mathrm{PSP}=$ peso seco de planta; $\mathrm{PFR}=$ peso fresco de raíz; $\mathrm{PSR}=$ peso seco de raíz; $\mathrm{RPP}=$ rendimiento por planta; $\mathrm{NFP}=$ número de frutos por planta; $\mathrm{SST}=$ sólidos solubles totales; $\mathrm{VitC}=$ vitamina $\mathrm{C} ; \mathrm{FT}=$ fenoles totales; $\mathrm{CAPs}=$ capsaicina; $\mathrm{NE}=$ número de esporas; \% $\mathrm{Col}=$ porcentaje de colonización; $\mathrm{SM}=\sin$ micorrizas; $\mathrm{CM}=$ con micorrizas.

\section{Conclusiones}

La malla azul con la menor RFA impactó positivamente en las variables agronómicas, de calidad y de respuesta al número de esporas y porcentaje de colonización. El ecotipo SNL influyó en variables de calidad como SST, Vit C y fenoles y NE y el RTZ en variables agronómicas y \% colonización. La inoculación con el consorcio de micorrizas produjo cambios favorables en los caracteres agronómicos y calidad del fruto de chile piquín.

\section{Literatura citada}

AOAC. 2000. Association of Official Analytical Chemists. Official Methods, Assoc. Off. Anal. Chem. Int. (AOAC), Arlington, VA, USA. 2000 p.

Arthurs, P. S.; Stamps, R. H and Giglia, F. F. 2013. Environmental modification inside photoselective Shadehouses. Hortscience. 48(8):975-979.

Ayala-Tafoya, F.; Yáñez-Juárez, Z. M. G.; Partida-Ruvalcaba, L.; Ruiz-Espinosa, F. H.; CamposGarcía H.; Vásquez-Martínez, O.; Velázquez-Alcaraz, T. de J. y Díaz-Valdés. 2015. Producción de pepino en ambientes diferenciados por mallas de sombreo fotoselectivo. Información Técnica Económica Agraria. 111(1):3-17. 
Bennet, D. J. and Kirby, G. W. 1965. Colorimetry of total phenolics with phosphomolibdicphpsphotungstic acid reagents. Ame. J. Enology and Viticulture. 16(3):144-158.

Cardona, G.; Peña, C. y Arcos, A. 2008. Ocurrencia de hongos formadores de micorriza arbuscular asociados a ají (Capsicum sp.) en la Amazonia colombiana. Agronomía Colombiana. 26(3):459-470.

Caruso, C.; Maucieri, C.; Barco, A.; Barbera, A. C. and Borin, M. 2018. Effects of mycorrhizal inoculation and digestate fertilisation on triticale biomass production using fungicidecoated seeds. Irish J. Agric. Food Res. 57(1):42-51.

Castellón-Martínez, E.; Carrillo-Rodríguez, J. C; Chávez-Servia, J. L. y Vera-Guzmán, A. M. 2014. Variación fenotípica de morfotipos de chile (Capsicum annuum L.) nativo de Oaxaca, México. Phyton. Inter. J. Exp. Bot. 83(2): 225-236.

García-Federico, A. S.; Montes-Hernández, J. A.; Rangel-Lucio, E.; García-Moya. y MendozaElos, M. 2010. Respuesta fisiológica de la semilla de chile piquín [Capsicum annuum var. glabriusculum (Dunal) Heiser \& Pickersgill] al ácido giberélico e hidrotermia. Rev. Mex. Cienc. Agríc. 1(2):203-216.

Gerdemann, J. W. and Nicolson, T. H. 1963. Spores of mycorrhizal Endogone species extracted from soil by wet sieving and decanting. Transactions of the British Mycological Society. 46(2):235-244.

Hernández-Verdugo, S.; González-Sánchez, R. A.; Porras, F.; Parra-Terraza, S.; Valdez-Ortiz, A.; Pacheco-Olvera, A. and López-España, R. G. 2015. Phenotypic plasticity of populations of wild chile (Capsicum annuиm var. glabriusculum) in response to light availability. Bot. Sci. 93(2):231-240.

Kelly, J. W. G.; Landhäusser, S. M. and Chow, P. S. 2015. The impact of light quality and quantity on root-to-shoot ratio and root carbon reserves in aspen seedling stock. New Forests. 46(4):527-545.

Kim, H. J.; Chen, F.; Wang, X. and Rajapakse, N. C. 2006. Effect of ethyl jasmonato on secundary metabolites of sweet basil (Ocimum basilicum L.) J. Agric. Food Chem. 54(6):2327-2332.

Koyama, A.; Pietrangelo, O.; Sanderson, L. and Antunes, P. M. 2017. An empirical investigation of the possibility of adaptability of arbuscular mycorrhizal fungi to new hosts. Mycorrhiza. 27(6):553-563.

McGonigle, T. P; Miller, M. H.; Evans, D. G.; Fairchild, G. L. and Swan, J. A. 1990. A new method which gives an objective measure of colonization of roots by vesiculararbuscular mycorrhizal fungi. New Phytol. 115(3):495-501.

Mujica, P. Y. 2012. Inoculación de hongos micorrízicos arbusculares (HMA) por dos vías diferentes en el cultivo del tomate (Solanum lycopersicum L). Cultivos Tropicales. 33(4):71-76.

Pagán, I.; Betancourt, M.; Miguel, J.; Piñero, D.; Fraile, A. and García-Arenal, F. 2010. Genomic and biological characterization of chiltepín yellow mosaic virus, a new tymovirus infecting Capsicum annuum var. aviculare in Mexico. Archives of Virology. 155(5):675-684.

Peixoto, B. V.; Pereira, C. C. M. A. e Ferreira, R. R. 2014. Emergência e crescimento inicial de Tabebuia heptaphylla (Vell.) Toledo en ambientes contrastantes de luz. Revista Árvore. 38(3):523-531.

Phillips, J. M. and Hayman, D. S. 1970. Improved procedures for clearing roots and staining parasitic and vesicular-arbuscular mycorrhizal fungi for rapid assessment of infection. Transactions of the British Mycological Society. 55(1):158-161. 
Quiñones-Aguilar, E. E.; López-Pérez, L; Hernández-Acosta, E; Ferrera-Cerrato, R y RincónEnríquez, G. 2014. Simbiosis micorrízica arbuscular y fuentes de materia orgánica en el crecimiento de Carica papaya L. Interciencia. 39(3):198-204.

Rodríguez, L. A.; Sánchez, R. D. L. C. and Silva, M. M. S. 2005. Effect of sunlight regimes on growth and yield of piquin pepper (Capsicum annuum L. var. aviculare). Rev. Chapingo Ser, Hortic.11(2):357-359.

Rueda, P. E. O.; Murillo, A. B.; Castellanos, C. T. J. L. G. H.; Tarazón, H. M. A.; Moreno, M. S. and Gerlach, B. L. E. 2010. Effects of plant growth promoting bacteria and mycorrhizal on Capsicum annuиm L. aviculare ([Dierbach] D'Arcy ans Eshbaugh) germination under stressing abiotic conditions. Plant Physiol. Biochem. 48(8):724-730.

Sánchez-Sánchez, A.; Salcedo-Martínez, S.; Mendoza-Villarreal, R.; Pinedo-Espinoza, J. and Moreno-Limón, S. 2018. Aislamiento e identificación de micorrizas arbúsculares (MA) asociadas a la rizósfera del chile piquín (Capsicum annuum var. aviculare L.). Investigación y Desarrollo en Ciencia y Tecnología de Alimentos. 3(4):86-91.

Shahak, Y.; Ratner, K.; Giller, Y. E.; Zur, N.; Or, E.; Gussakovsky, E. E. and Greenblat-Avron, Y. 2006. Improving solar energy utilization, productivity and fruit quality in orchards and vineyards by photoselective netting. In: XXVII International Horticultural CongressIHC2006: International Symposium on Enhancing Economic and Environmental. 772(1):65-72.

Wei, Z. F.; Luo, M.; Zhao, C. J.; Li, C. Y.; Gu, C. B.; Wang, W.; Zu, Y. G.; Efferth, T. and Fu, Y. J. 2013. UV-Induced changes of active components and antioxidant activity in postharvest pigeon pea [Cajanus cajan (L.) Millsp.] leaves. J. Agric. Food Chem. 61(6):1165-1171. 